19 Hill PD, Aldag JC, Chatterton RT Jr. Breastfeeding experience and milk weight in lactating mothers pumping for preterm infants. Birth 1999;26:233-8.

20 Meier PP. Breastfeeding in the special care nursery. Prematures and infants with medical problems. Pediatr Clin North Am 2001;48:425-42.

21 Seema MD, Patwari AK, Satyanarayana L. Relactation an effective intervention to promote exclusive breastfeeding. J Trop Pediatr 1997:43:213-6.

22 da Silva OP, Knoppert DC, Angelini MM, et al. Effect of domperidone on milk production in mothers of premature newborns: a randomized double-blind placebo-controlled trial. CMAJ 2001;164:17-21.
23 Kent JC, Ramsay DT, Doherty D, et al. Response of breasts to different stimulation patterns of a electric breast pump. J Hum Lact 2003:9:157-65.

24 Hurst NM, Valentine CJ, Refro L, et al Skin-to-skin holding in the neonatal intensive care unit influences maternal milk volume. J Perinatol 1997;17:213-7.

25 Whitelaw A, Heisterkamp G, Sleath K. Skin to skin contact for very low birth weight infants and their mothers. Arch Dis Child 1988:63:1377-81.

26 Bier JB, Ferguson AE, Morales Y, et al. Skin-to-skin contact improves physiologic states of breast-fed low birth-weight (LBW) infants (abstract). Pediatr Res 1995; 37:103.

27 Fewtrall MS, Loh KL, Blake A, et al. Randomised, double blind trial of oxytocin nasal spray in mothers expressing breast milk for preterm infants. Arch Dis Child Fetal Neonatal Ed 2006;91:F169-74.

28 Arnold L. Recommendations for collection, storage and handling of a mother's milk for her own infan in a hospital setting, 3rd edn. Sandwich, MA: Human Milk Banking Association of North America 1999.

29 Jones E, Dimmock PW, Spencer SA. A randomised controlled trial to compare methods of milk expression following preterm delivery. Arch Dis Child Fetal Neonatal Ed 2001;85:F91.

30 Jones $\mathbf{E}$, Jones $\mathrm{P}$, Dimmock PW, et al. Evaluating preterm breastfeeding training. Pract Midwife 2004;7:19-24.

\title{
Optical imaging of the neonatal brain
}

\section{Topun Austin}

\section{Optical systems could be valuable tools for assessing cerebral function at the cotside}

$\mathrm{T}$ he newborn brain is vulnerable to a variety of insults with potentially lifelong consequences. As our understanding of the mechanism of brain injury improves and new therapies are developed to prevent or minimise brain injury, new non-invasive methods are required to assess cerebral function at the cotside.

The application of near-infrared spectroscopy (NIRS) for continuous monitoring of cerebral haemodynamics and oxygenation non-invasively was first reported by Jobsis in 1977. ${ }^{1}$ Since that time NIRS has become an effective research tool for studying infant cerebral haemodynamics and oxygenation. ${ }^{23}$ NIRS exploits the relative transparency of biological tissue to near-infrared light (700-1000 nm), and the wavelengthdependent absorption characteristics of haemoglobin, which vary with oxygenation. By monitoring the intensity of light passing through brain tissue at two or more wavelengths, observed changes in attenuation can be converted into changes in the cerebral concentrations of oxyhaemoglobin and deoxyhaemoglobin.

\section{FUNCTIONAL NIRS}

An obvious application of NIRS is the investigation of localised changes in oxyhaemoglobin and deoxyhaemoglobin resulting from functional activation of the cerebral cortex. Increases in local cerebral blood flow (CBF) are manifested by a rise in oxyhaemoglobin and a fall in deoxyhaemoglobin whereas oxygen consumption during activation results in a decrease in oxyhaemoglobin and an increase in deoxyhaemoglobin. The balance between local perfusion and consumption can be investigated with NIRS

The first functional studies in the newborn with optical techniques used conventional single source-detector systems to measure overall changes in oxygenation over a particular area of the head. Meek et al reported that, in response to a visual stimulus, there was an increase in both oxyhaemoglobin and deoxyhaemoglobin over the occipital lobe. ${ }^{4}$ This finding was consistent with those of studies in infants using functional MRI and contrasted with the response in adults, in whom the increase in regional perfusion greatly exceeds the increase in local consumption, resulting in an increase in oxyhaemoglobin and decrease in deoxyhaemoglobin. ${ }^{5}$ The maturation of this response seems to depend on the type of stimulus and the state of wakefulness of the infant as well as their age, and it is currently an area of active research using optical topography. ${ }^{6-10}$

Bartocci et al investigated the response to olfactory stimuli in the newborn: they found a significant increase in oxyhaemoglobin measured over the frontal cortex in response to colostrum which was inversely related to postnatal age. ${ }^{11}$ Neonates were also able to differentiate between pleasant and unpleasant odours. ${ }^{12}$ Recently the haemodynamic response to pain has been investigated using NIRS. ${ }^{13}{ }^{14}$ Slater et al found that preterm infants as young as 25 weeks' gestation show a cortical response following a heel lance. The response was markedly greater in infants who were awake, indicating the importance of behavioural state in the response to pain.

\section{OPTICAL TOPOGRAPHY}

The single source-detector pair studies outlined above obtain measurements over a relatively large volume of the brain and are therefore susceptible to inaccuracies in both localisation and quantification of the response. Optical imaging is therefore a natural extension of efforts to address these issues.

The most straightforward approach is optical topography, which involves acquiring multiple reflectance measurements at small source-detector separations from the surface of the head simultaneously or in rapid succession (fig 1A). By keeping the separations small it is possible to make measurements of rapid changes in haemodynamics associated with functional activation. The penetration of light is limited and so will reflect changes in oxygenation within the cerebral cortex. Several groups have developed custom-built multichannel topographic imaging systems demonstrating the potential of this technique to assess brain function at the cotside. ${ }^{15}{ }^{16} \mathrm{~A}$ commercially available optical topography system (Hitachi ETG-100 optical topography system, Hitachi Medical Corporation, Tokyo, Japan) has been used to investigate the response of neonates to a variety of stimuli, including visual, auditory and somatosensory. ${ }^{81718}$ Pena et al investigated the response of neonates to speech in the first few days of life. ${ }^{18}$ They found the greatest change in regional blood volume, over the left temporal lobe, was in response to the mother's speech, compared with "reversed speech", illustrating the remarkable ability of the human brain for complex neural processing in the first days of life.

\section{OPTICAL TOMOGRAPHY}

In contrast to optical topography, the aim of optical tomography is to obtain a twodimensional slice or a three-dimensional image of the whole brain. The sensitivity to deep tissue requires measurements across the head, and consequently 


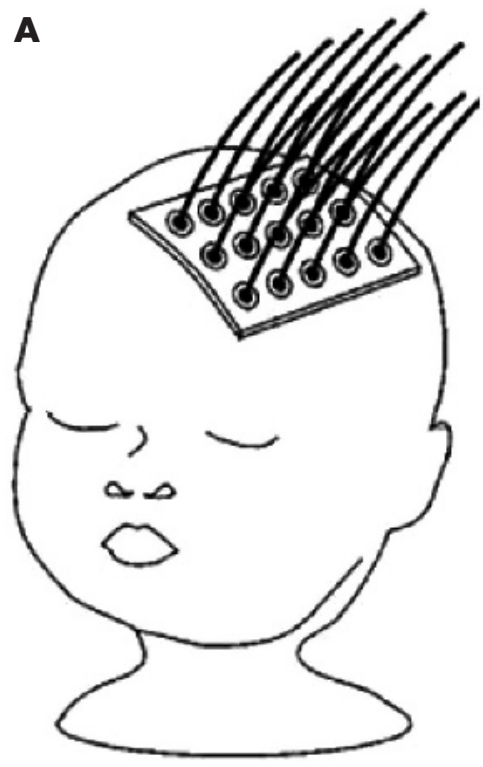

Figure 1 (A) Optical topography: the close proximity of optical fibres allow rapid measurements to be obtained from the surface of the brain. (B) Three-dimensional optical tomography: interrogation of the whole volume of the brain is possible by placing optical fibres around the head.

transmitted light must be interrogated over periods of several seconds or longer for each source to obtain adequate signal (fig 1B). Although this inhibits the display of fast haemodynamic phenomena associated with functional activation, unless averaged over repeated stimuli, this technique is able to obtain information on oxygenation in deeper areas of the brain. The greatest challenge with optical tomography is developing adequate image reconstruction algorithms which can account for the overwhelming scattering of light as it passes through the head.

\section{Static imaging of the infant brain}

Two optical tomography systems have been used to date to image the neonatal brain, both based on the measurement of times of flight of photons travelling across the head. The distribution of photon flight times is unique for each source-detector pairing and provides information on the light absorbing and scattering characteristics of the tissue being interrogated.

The first two-dimensional tomographic images of the brain were demonstrated by Benaron et al, who developed an imaging system that measures photon flight times between points arranged around the circumference of the head. ${ }^{19} 20$ Images representing a transverse slice across the brain were reconstructed using a relatively straightforward backprojection method. ${ }^{21}$ Scans of infants at a variety of gestational ages successfully showed intracranial haemorrhage and a focal region of low oxygenation after acute stroke. $^{19} 2022$ A major drawback of this

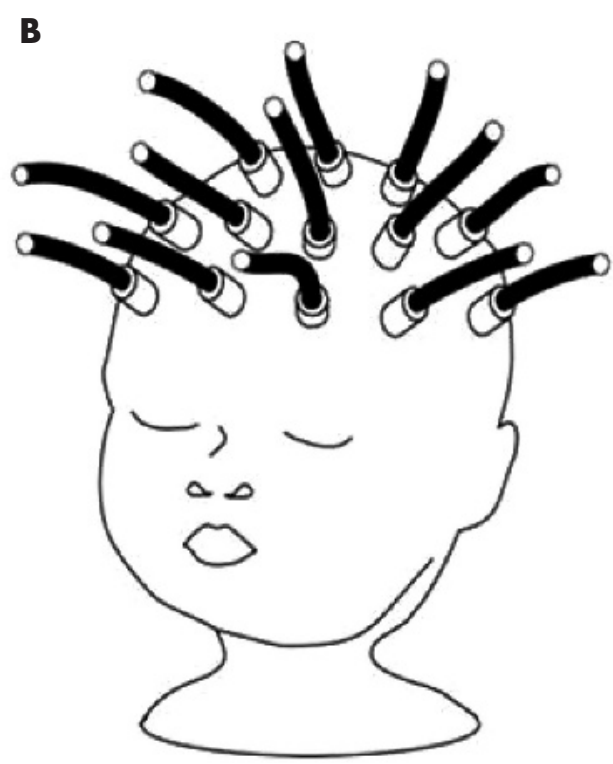

system was the acknowledged simplicity of the image reconstruction algorithm, which ignored the inherent three-dimensional nature of photon migration in tissues, and the highly heterogeneous nature of the infant head.

To address this issue an iterative nonlinear algorithm has been developed at University College London (UCL). ${ }^{23}$ The algorithm compares the measured data with the simulated data derived from a computer model of the infant brain, and updates the model iteratively until a satisfactory match is achieved. Data have been acquired using a 32-channel time resolved system, designed and built at the Biomedical Optics Research Laboratory, UCL. ${ }^{24}$ The instrument illuminates the infant head with picosecond pulses of light at two wavelengths $(780 \mathrm{~nm}$,
$815 \mathrm{~nm})$. Reconstruction images of the internal absorption properties can then be used to generate images of regional cerebral blood volume and regional tissue oxygen saturation. ${ }^{25}$

Initial studies on premature infants used custom-made, foam-lined, plastic helmets whose dimensions were based on a series of measurements acquired from digital photographs of each infant prior to the study. More recent studies have used an adaptable helmet that can accommodate head sizes of infants from 24 weeks' gestation to term. A complete dataset can be acquired in 10 minutes. Because the system measures the times of flight of transmitted photons it is less sensitive to movement artefact than conventional near-infrared instruments that only measure transmitted intensity. To date nearly 40 infants have been scanned using the UCL system, with roughly twothirds of the scans yielding data sufficient for reliable image reconstruction. Failure to obtain images has been due to a variety of factors, such as infant movement, poorly fitting helmet or instrumental instability. The first successful images were reconstructed from a 30 -week preterm infant with a large unilateral intraventricular haemorrhage ${ }^{26}$; this was followed by images obtained on an infant requiring mechanical ventilation. By making appropriate changes to the ventilator settings a unique series of images revealed regional changes in cerebral blood volume and oxygenation. ${ }^{27}$

Images of regional cerebral blood volume and regional tissue oxygen saturation from healthy infants show considerable heterogeneity, with a reduction in blood volume and oxygenation in the more central regions of the brain. ${ }^{28}$ This is consistent with studies of regional perfusion using single photon emission computed tomography, demonstrating the vulnerability of the periventricular

\section{A}
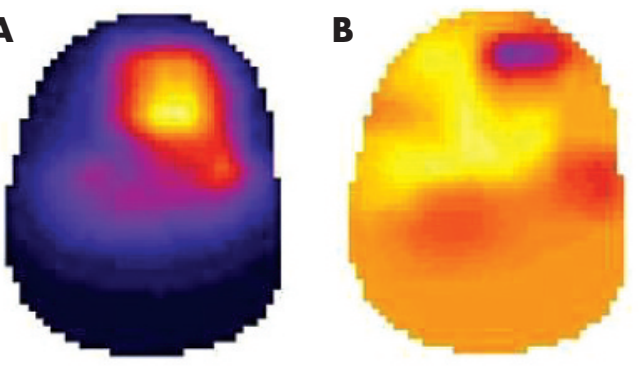

$0 \quad 40 \% 0$

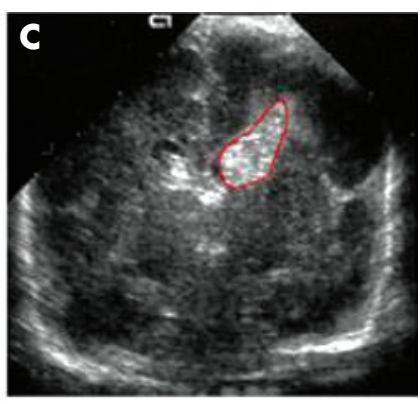

$85 \%$
Figure 2 Coronal sections of infant brain images of (A) regional blood volume, (B) regional oxygen saturation and $(C)$ the corresponding ultrasound scan. Reprinted from Neurolmage, 31, Austin $T$, Gibson AP, Branco $G$, et al. Three-dimensional optical imaging of blood volume and oxygenation in the preterm brain, figure 5, p. 1431, ( 2006, with permission from Elsevier. 

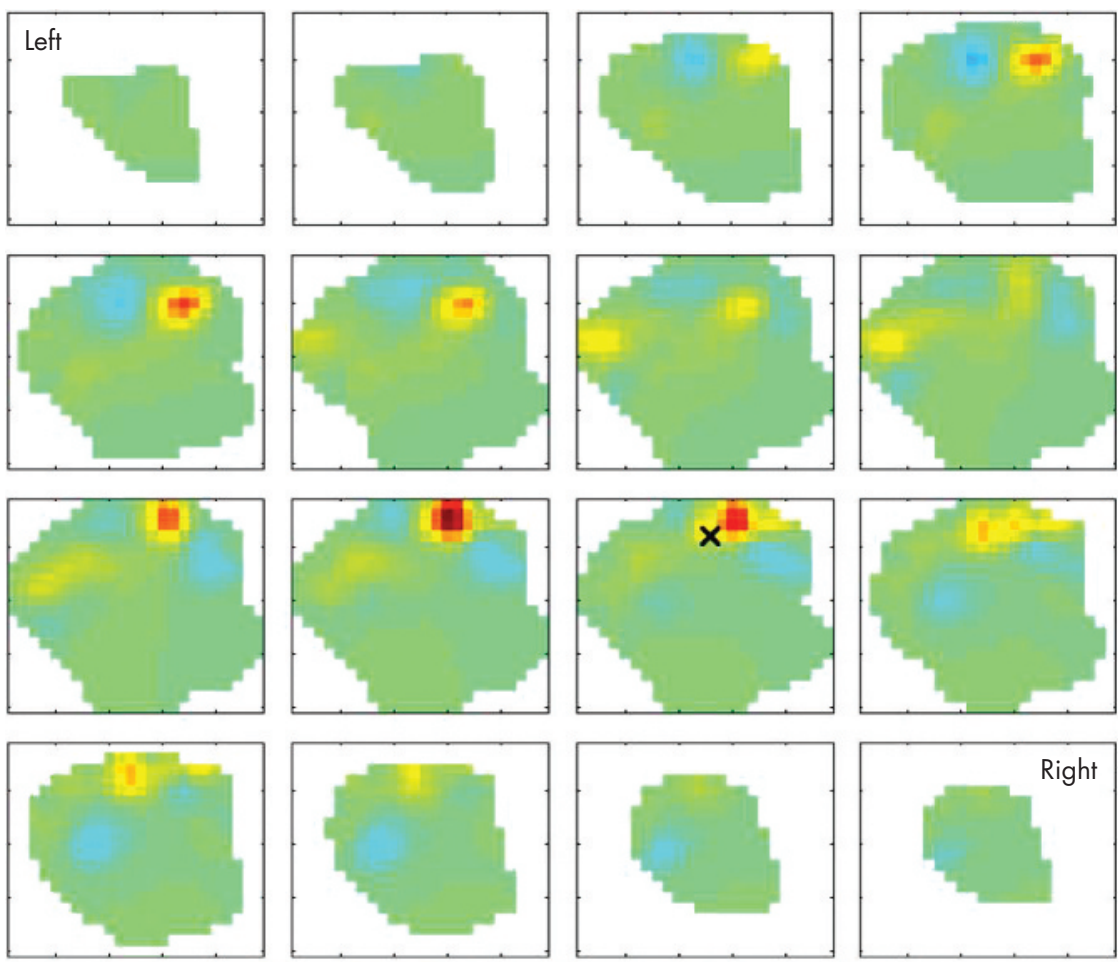

$-38$

$38 \mu \mathrm{M}$

Figure 3 Sagittal slices across three-dimensional image of change in total haemoglobin concentration in an infant brain due to passive movement of the left arm, showing an increase near the estimated position of the right motor cortex (denoted by the cross). Reprinted from Neurolmage, 30, Gibson AP, Austin T, Everdell NL, et al. Three-dimensional whole-head optical tomography of passive motor evoked responses in the neonate, figure 4, p. 526, ${ }^{\circ} 2006$, with permission from Elsevier.

white matter to hypoxic-ischaemic injury. ${ }^{29}$ Figure 2 shows coronal slices from threedimensional images of regional cerebral blood volume and regional tissue oxygen saturation from a preterm infant with a left-sided intraventricular haemorrhage and haemorrhagic parenchymal infarct. A distinct area of desaturated haemoglobin on the left side is seen ( $10 \%$ compared with $62 \%$ on the contralateral side). Although more lateral and superficial than the position indicated by the ultrasound scan this is consistent with a resolving blood clot. An ischaemic penumbra surrounding haemorrhagic lesions has been described both in adults and infants and optical tomography will be the ideal tool to explore this concept further. ${ }^{30} 31$

\section{Three-dimensional imaging of functional activation}

We have recently obtained the first threedimensional images of the entire neonatal head during motor evoked responses. $^{32}$ Data were acquired during bilateral passive arm movement, repeated for 12 source positions. A composite image was reconstructed for each infant and demonstrated an increase in total haemoglobin concentration in an area consistent with the contralateral motor and sensory cortex (fig 3). The amplitude of the measured increase was $25 \mathrm{mM}-$ greater than the equivalent change observed using NIRS and optical topography. ${ }^{48}$ This is likely to reflect the improved spatial resolution and hence reduced partial volume effect of the three-dimensional imaging system.

\section{Current challenges with optical tomography}

The biggest challenges in three-dimensional optical tomography are to improve quantitation and spatial resolution. Although the spatial resolution of optical tomography is not likely to match that of MRI, CT or ultrasound, it is probably comparable with positron emission tomography.

Improved resolution is possible by incorporating prior anatomical information from generic MRI scans of newborn infants and this approach is being actively pursued by our group at UCL. The current instrument is large and requires a team of physicists, engineers and computer scientists to operate and reconstruct the images. Work is underway at developing a smaller and faster instrument with data collection and reconstruction software that can be used by clinicians. Although difficult to predict the cost of a commercial instrument, it will probably be similar to that of other portable imaging tools, such as ultrasound.

\section{FUTURE DEVELOPMENTS IN OPTICAL IMAGING}

Over the past 25 years the clinical application of NIRS has been frustratingly slow. ${ }^{33}$ The challenge is to develop optical imaging systems into clinically useful cotside tools. Three-dimensional optical tomography represents a major advance in this field. Unlike functional MRI, optical images can be acquired in unsedated infants at the cotside; by measuring both oxyhaemoglobin and deoxyhaemoglobin independently it is possible to distinguish the increased oxygen extraction due to brain activation from changes in regional blood volume due to local vasodilatation. Measurements from deep inside the brain will allow cerebral maturation in the preterm brain to be investigated.

Optical imaging currently uses compounds that absorb natural light in the brain; however, it is equally sensitive to exogenous contrast agents. ${ }^{34}$ One can expect, with the development of new molecular optical imaging markers, optical tomography will be able to provide unique physiological images in vivo. This would represent a remarkable advance in the brain-oriented care of the newborn.

\section{ACKNOWLEDGEMENTS}

The author would like to thank the following members of the Biomedical Optics Research Laboratory and Institute for Women's Health at UCL: Professor Jem Hebden, Dr Adam Gibson, Professor John Wyatt and Dr Judith Meek.

The work carried out at UCL was funded by grants from the Medical Research Council, Wellcome Trust and Engineering and Physics Research Council with support from University College London Hospitals NHS Foundation Trust.

Arch Dis Child Fetal Neonatal Ed 2007;92:F238-F241.

doi: $10.1136 /$ adc. 2006.103846

Correspondence to: Dr Topun Austin, Perinatal Brain Injury and Repair Group, Institute for

Women's Health, University College London, 4th Floor, Rayne Building, 5 University Street, London WC1E 6JJ, UK; topunaustin@doctors.org.uk

Accepted 23 January 2007

Competing interests: None declared.

\section{REFERENCES}

1 Jobsis FF. Noninvasive, infrared monitoring of cerebral and myocardial oxygen sufficiency and circulatory parameters. Science 1977; 198:1 264-7.

2 Wyatt JS, Cope M, Delpy DT, et al. Quantification of cerebral oxygenation and haemodynamics in sick newborn infants by near infrared

spectrophotometry. Lancet 1986;8515:1063-6. 
3 Yoxall CW, Weindling AM, Dawani NH, et al. Measurement of cerebral venous oxyhaemoglobin saturation in children by near-infrared spectroscopy and partial jugular venous occlusion. Pediatr Res 1995;38:319-23.

4 Meek JH, Firbank M, Elwell CE, et al. Regional haemodynamic responses to visual stimulation in awake infants. Pediatr Res 1998;43:840-3

5 Born P, Leth H, Miranda MJ, et al. Visual activation in infants and young children studied by functional magnetic resonance imaging. Pediatr Res 1998:44:578-83.

6 Hoshi Y, Kohri S, Matsumoto Y, et al. Hemodynamic responses to photic stimulation in neonates. Pediatr Neurol 2000;23:323-7.

7 Chen $Y$, Zhou S, Xie C, et al. Preliminary evaluation of dual wavelength phased array imaging on neonatal brain function. J Biomed Optics 2000;5:194-200.

8 Taga G, Asakawa K, Maki A, et al. Brain imaging in awake infants by near-infrared optical topography. Proc Natl Acad Sci U S A, 2003;100:10722-7.

9 Taga G, Asakawa K, Hirasawa K, et al. Hemodynamic responses to visual stimulation in occipital and frontal cortex of newborn infants: a near-infrared optical topography study. Early Hum Dev 2003;75:S203-10.

10 Kusaka T, Kawada K, Okubo K, et al. Noninvasive optical imaging in the visual cortex in young infants. Hum Brain Mapp 2004;22:122-32.

11 Bartocci M, Winberg J, Ruggiero C, et al. Activation of olfactory cortex in newborn infants after odor stimulation: a functional near-infrared spectroscopy study. Pediatr Res 2000;48:18-23.

12 Bartocci M, Winberg J, Papandieck G, et al. Cerebral haemodynamic response to unpleasant odours in the preterm newborn measured by near-infrared spectroscopy. Pediatr Res 2001;50:324-30.

13 Batrotcci M, Bergqvist LL, Lagercrantz H, et al. Pain activates cortical areas in the preterm newborn brain. Pain 2006;122:109-17.
14 Slater R, Cantarella A, Gallella S, et al. Cortical pain responses in human infants. J Neurosci 2006;26:3662-6.

15 Hintz SR, Benaron DA, Siegal AM, et al. Bedside functional imaging of the premature infant brain during passive motor activation. J Perinatal Med 2001;29:335-43.

16 Kotilahti K, Nissilä I, Huotilainen M, et al. Bilatera hemodynamic responses to auditory stimulation in newborn infants. Neuroreport 2005;16:1373-7.

17 Isobe K, Kasuka T, Nagano K, et al. Functional imaging of the brain in sedated newborn infants using near infrared topography during passive knee movement. Neurosci Lett 2001 ;299:221-4.

18 Pena M, Maki A, Kovacic D, et al. Sounds and silence: an optical topography study of language recognition at birth. Proc Natl Acad Sci U S A 2003; 100:11702-5.

19 Hintz SR, Benaron DA, van Houten JP, et al. Stationary headband for clinical time of flight optical imaging at the bedside. Photochem Photobiol 1998;68:361-9.

20 Benaron DA, Hintz SR, Villringer A, et al. Noninvasive functional imaging of human brain using light. J Cereb Blood Flow Metab 2000;20:469-77.

21 Benaron DA, Ho DC, Spilman S, et al. Non-recursive linear algorithms for optical imaging in diffusive media. Adv Exp Med Biol 1994;361:215-22.

22 Hintz SR, Cheong W-F, van Houten JP, et al. Bedside imaging of intracranial haemorrhage in the neonate using light: comparison with ultrasound, computed tomography and magnetic resonance imaging. Pediatr Res 1999;45:54-9.

23 Arridge SR, Schweiger M. Image reconstruction in optical tomography. Philos Trans R Soc Lond B Biol Sci 1997;352:717-26.

24 Schmidt FEW, Fry ME, Hillman EMC, et al. A 32 channel time-resolved instrument for medical optical tomography. Rev Sci Inst 2000;71:256-65.
25 Hillman EMC. Experimental and theoretical investigations of near-infrared tomographic imaging methods and clinical applications. PhD imaging methods and clinical applications.
Thesis, University of London, 2002. http:// www.medphys.ucl.ac.uk/research/borg/ homepages/beth/thesis/ Elizabeth Hillman PhDthesis2002.pdf

26 Hebden JC, Gibson A, Yusof R, et al. Three dimensional optical tomography of the premature infant brain. Phys Med Biol 2002;47:4155-66.

27 Hebden JC, Gibson A, Austin T, et al. Imaging changes in blood volume and oxygenation in the newborn infant brain using three-dimensional optical tomography. Phys Med Biol 2004;49:1 $117-30$.

28 Austin T, Gibson AP, Branco G et al. Threedimensional optical imaging of blood volume and oxygenation in the preterm brain. Neurolmage 2006;31:1426-33.

29 Borch K, Greisen G. Blood flow distribution in the normal human preterm brain. Pediatr Res 1998:43:28-33.

30 Powers WJ, Press GA, Grubb RL, et al. The effect of hemodynamically significant carotid artery disease on the hemodynamic status of the cerebral circulation. Ann Intern Med 1987; 106:27-34.

31 Volpe JJ, Herscovitch P, Perlman JM, et al. Positron emission tomography in the asphyxiated term newborn: parasagittal impairment of cerebral blood flow. Ann Neurol 1985; 17:287-96.

32 Gibson AP, Austin T, Everdell NL, et al. Three dimensional whole-head optical tomography of passive motor evoked responses in the neonate. Neurolmage 2006;30:521-8.

33 Greisen G. Is near-infrared spectroscopy living up to its promises? Semin Fetal Neonatal Med 2006;11:498-502.

34 Kusaka T, Isobe K, Nagano K, et al. Estimation of regional cerebral blood flow distribution in infants by near-infrared topography using indocyanine green. Neurolmage 2001;13:944-52.

\section{Prescribing home oxygen}

\section{Gaynor Harrison, Ben Shaw}

\section{The new oxygen service-providing consistency throughout the UK}

$\mathrm{H}$ ome oxygen therapy services have a vital role in supporting children with breathing difficulties, including those with long-term medical conditions such as chronic lung disease of the newborn, pulmonary interstitial fibrosis, neurodisability and cystic fibrosis. ${ }^{1}$ This article discusses the practicalities of prescribing oxygen for children who require it in the home.

Until recently, oxygen was prescribed by the patient's general practitioner (GP), with a supplier providing the oxygen concentrator service and local pharmacies supplying oxygen cylinders to patients in their homes. Liquid oxygen was only available following application to the primary care trust (PCT) for funding. This resulted in variations and inconsistencies throughout the UK in the way home oxygen was prescribed, used and delivered. In 2003, the Department of Health $^{2}$ announced plans to modernise the domiciliary oxygen service to improve patient access to a wider range of modern technologies supporting patients' clinical care and other needs. The aim was to improve quality of life, ambulatory oxygen provision, holiday provision in the UK and safety. From February 2006, following the development of new service specifications and a competitive tendering process, four companies (Air Products, Allied Respiratory, Linde Gas and BOC) were awarded contracts to provide this service across 11 regions in England and Wales. Domiciliary oxygen is now provided by a single contractor in each home oxygen service region and all modalities are available, including liquid oxygen. Air Products is the provider to most of the regions in England and Wales.

Once a decision has been made for a child to be discharged on supplemental oxygen, a home oxygen consent form
(HOCF) is signed by the parents (box 1 , step 1). A home oxygen order form (HOOF) is completed (in large trusts this is done by a designated healthcare professional who may be a doctor or clinical nurse specialist) (box 1, step 2; note than some areas it is still not clear who the clinical lead is - they may be an employee of the PCT or a lead clinician (doctor or nurse) in a trust). The supplier confirms that they have received the fax (box l, step 3) and are dealing with the order. The oxygen company (box 2, step 4) then contacts the family directly to installs the oxygen equipment in the child's home.

Depending on the details given on the HOOF, the oxygen supplier will install the oxygen within three working days, the next day if the patient is being discharged from hospital or within four hours if required as an emergency. The HOCF/ HOOF and other key documents and guidance can be downloaded from the primary care contracting website. ${ }^{3}$

\section{OXYGEN EQUIPMENT}

Oxygen suppliers have a variety of oxygen equipment (fig 2), ${ }^{45}$ including oxygen concentrators, cylinders with integrated valves, conservers (not used for oxygen flows less than $1 \mathrm{l} / \mathrm{min}$ and not generally used for children), liquid oxygen and associated consumables (nasal cannulae/ masks/portable cylinder carry bags etc). If a concentrator machine breaks down they 